\title{
3D Visualization of Large Digital Elevation Model (DEM) Data Set
}

\author{
Min Sun ${ }^{1}$, Yong Xue ${ }^{2,3}$, Ai-Nai Ma ${ }^{1}$ and Shan-Jun Mao ${ }^{1}$ \\ ${ }^{1}$ Institute of Remote Sensing and Geographic Information System, Peking University, \\ Beijing 100871, China \\ \{Sm6902@263.net, maainai@pku.edu.cn\} \\ ${ }^{2}$ Laboratory of Remote Sensing Information Sciences, Institute of Remote Sensing \\ Applications, Chinese Academy of Science, PO Box 9718, Beijing 100101, China \\ \{yxuedirsa.irsa.ac.cn\} \\ ${ }^{3}$ School of Informatics and Multimedia Technology, University of North London, 166-220 \\ Holloway Road, London N7 8DB, UK \\ $\{y \cdot x u e @ u n l \cdot a c \cdot u k\}$
}

\begin{abstract}
With the rapid development of Earth observation technologies, raster data has become the main Geographic Information System (GIS) data source. GIS should be able to manage and process huge raster data sets. In this paper, the problem of 3-dimensional visualization of huge DEM data sets and its corresponding images data sets in GIS was addressed. A real-time delamination method based on raster characteristics of DEM and image data has been developed. A simple geometry algorithm was used to implement dynamic Level of Division (LOD) delamination of DEM and image data and to realize realtime 3-dimensional visualization of huge DEM and image data set based on RDBMS management.
\end{abstract}

\section{Introduction}

In recent years, Earth observation technologies such as remote sensing, SAR, airborne laser scan, and Photogrammetry, have got a rapid progress. The amount of the data captured by these technologies is increasing in geometric series. Raster data would become the main GIS data source. Many research works on huge raster data management have been done, e.g., Fang et al. [2] developed a GeoStar software to manage multi-resolution image data using pyramid style in file system. The software can be used to seamlessly manage multi-scale and multi-resolution image data. Nebiker [6] proved that high-performance relational database management systems could be used to manage conceptually unlimited amounts of raster data. But how to perform data operation on the base of huge data management is still a difficult question, e.g. 3-dimensional visualization of huge DEM data and its image data.

Most 3-dimensional DEM data visualization methods and theories are used for single DEM data block. They are emphasized on how to simplify and efficiently manage the terrain data in order to realize visualization in high speed with high precision. Many algorithms introduced in order to simplify the data were usually 
established on the data structure which were used to manage the terrain data, e.g., the data structure based on quadtree. These methods pretreated DEM data before visualization. It not only effects real-time rendering speed, but also limits visual data area, therefore, its difficult to realize visualization of huge DEM data set in real time.

Some typical works on 3-dimensional visualization of DEM data are: Cignoni et al. [1] expressed multi-resolution DEM data using hierarchical TIN structure. This method needs great calculation and it is difficult to realize real-time visualization. Lindstrom [5] put forward a real-time, continuous LOD rendering method. The basic idea of this method is to manage DEM data using quadtree data structure. When the DEM data block is huge, the quadtree data structure itself would need a huge memory space (e.g.: $20480 \times 20480$ grid DEM data, about $400 \mathrm{Mb}$, if $4 \times 4$ grid form a node, each node occupies 16 bits, then it would cast $400 \mathrm{Mb}$ memory space). Despite the fact that this method could simplify a huge amount of data, it still needs a large amount of data in order to render details in high precision. The algorithm have an great effect on rendering speed, so it is difficult to deal with huge DEM data sets. Hoppe [3] also put forward an algorithm that could be used to dynamically calculate LODs based on viewpoint. But this method is still difficult to deal with huge DEM data real-time rendering. All these works are based on TIN structure. They need great calculation in real-time rendering and pretreatment, and also high hardwires configure. They are suitable to run on graphic workstations.

Comparing with TIN structure, regular grid structure is much simple and DEM usually was expressed using regular grid in practice. A common style is point matrix of raster structure, saving in image format. Research works on 3D visualization of DEM data based on regular grid are: Kofler [4] developed a method combining LOD and R-tree structure. This method used R-tree instead of quadtree. It would be more difficult for this method used to realize huge DEM data sets 3D visualization. Wang et al. [7] put forward a method based on quadtree structure and simplify the data depending viewpoint position. As this method used quadtree structure to manage DEM data, it used a lot of memory and it is difficult for huge DEM data sets visualization.

So far, there is no one method which could be used to solve the problem of huge DEM data sets real-time visualization properly.

\section{Real-time LOD delamination based on regular grid}

\subsection{Regular grid division}

Regular grid structure is easy to process and DEM data formats are mainly using regular grid. The basis of our method for huge DEM data sets real-time visualization is to express DEM data using regular grid. DEM regular grid format is in 2dimensional matrix. As its length and width is known, one dimension array, e.g., $p[n]$, is used normally. If a plane's original point position is $(0,0)$, the length and width of DEM are $\mathrm{s}$ and $\mathrm{t}$, the height value of a random point $(i, j)$ is $p[i * t+j]$. This concise 
structure of regular grid actually manages DEM data very good. There is no need to manage it using extra structure.

According to LOD idea, terrain areas in far viewpoint would not need same precision as that in near viewpoint. So we could use viewpoint distance to divide grid LOD delamination in order to improve visualization speed.

In Figure 1, $e$ is the viewpoint position, $a$ is observation angle, $b$ is the angle between sightline and vertical direction and $p$ is the center point of view area on DEM. Then the dash-line circle marked area needs high rendering precision LOD. For the convenience of calculation, we use the square instead of this circle. The different LOD areas are expressed by nested square area. Based on this simple thinking, we could easily get LOD division of any random time and random viewpoint position.
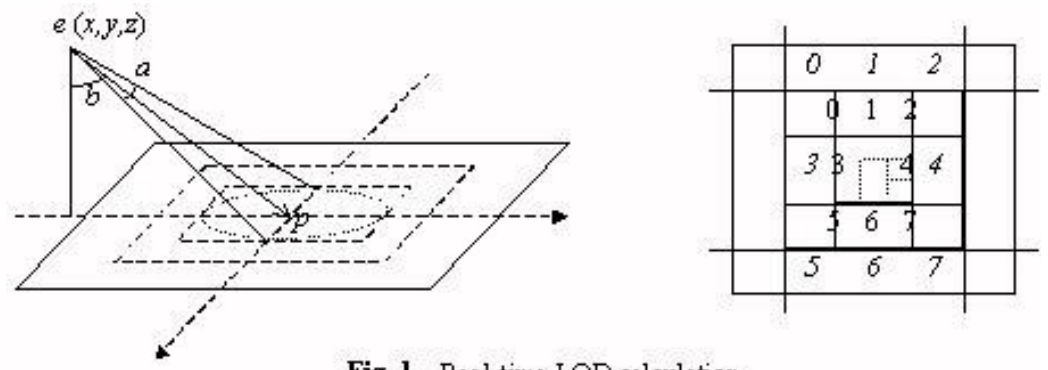

Fig. 1. Real-time LOD calculation

Right picture in Figure 1 shows the principle of LOD repetition calculation. Any LOD area could be consider as combination of 8 blocks. Its size could be calculated using formula $D=m \times 2^{n}, m$ expresses the size of first LOD area where the viewpoint is in. The value of $m$ could change with the distance from $e$ to $p$.

Regular grid data usually results in interposition from discrete points on plane. It possess a huge number of abundance data. Adjacent points have close height values, so we could simplify the DEM and its corresponding image data by resample method to get LOD real-time rendering data. For the first LOD area around viewpoint $p$, resample one in two points on the base of original DEM and image data, for second LOD area resample one in four points, for $n$ LOD area resample interval use $2^{n}(n=1$, $2, \ldots)$.

Figure 2 shows the above resample process. If we divided according $m \times 2^{n}$, then the actual rendering DEM grid number $t m$ in real-time is:

$$
\begin{aligned}
& t m=(2 m / 2)^{2}+\left(4 \times\left[\left(m \times 2^{n-1} / 2^{n}\right)^{2}+\right.\right. \\
& \left.\left.\left(2 m+m \times 2^{n-1}\right) \times m \times 2^{n-1} / 2^{n}\right]\right) \\
& =m^{2}+m^{2}\left(3+2^{n+1}\right) \\
& =\left(3 n+2^{n+2}\right) m^{2} .
\end{aligned}
$$

The actual DEM size is:

$\left[2 \times\left(m+\ldots+2^{n-1} m\right)\right] \times[2 \times(m+$

$\left.\left.\ldots+2^{n-1} m\right)\right]$

$=\left(2^{n+1}-2\right)^{2} m^{2}$

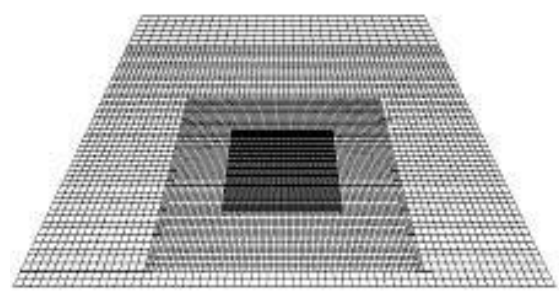

if we let $m=64 n=5$, then real-time rendering grid number is: $143 \times 64^{2}=$ 585,728 . 
While DEM size is: $\left(2^{6}-2\right)^{2} \times 64^{2}=(62 \times 64)^{2}=3968 \times 3968$

The actual DEM grid number is $15,745,024$, the simplify process discards $96.3 \%$ original data.

\subsection{Further simplify strategies}

For personal computer environment, it is still difficult to render in real-time although more than ten millions grids have been simplified to less than one million. As above simplification does not consider the observation angle, the distance from viewpoint to observation point and view area, these parameters could be used to do further simplification.

Assume that $L O D_{i}$ projection value on screen is $g$. If $g<=d$ ( $d$ is an environment parameter given by user through system, it is at least large than 1 pixel). That is, when the projection of $L O D_{i}$ on screen is less than or equal to $d$ pixels, $L O D_{i}$ no longer be rendered. In this way, far view area could be controlled in real-time rendering. In Figure $3, A B$ is the width of $L O D_{i}$,

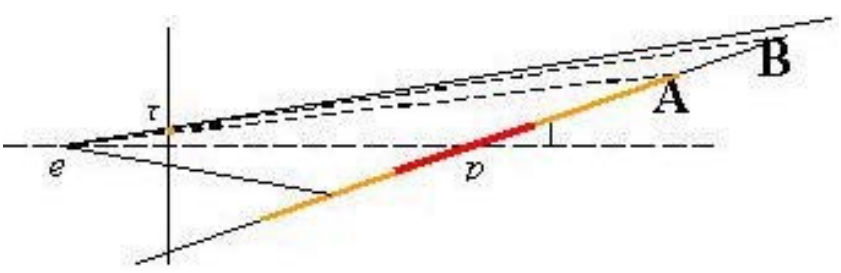
and its projection value on screen is $\hat{o}$. Whenôis too small to be noticed, there is no need to render $A B$. Therefore, the fact of this strategy is to controlled DEM visual area so as to decrease DEM rendering data in real time.

Also, assume that the present screen width is $r$ and $L O D_{i}$ projection width on screen is $f$. If $r>f, L O D_{i}$ will be replaced by $L O D_{i+l}$, contrarily $L O D_{i+l}$ is replaced by $L O D_{i}$. In such LOD exchange process, firstly the system does not keep so much DEM blocks which are read from the database. Secondly the width of view area could cut off at least $50 \%$ grid number in real-time rendering.

In Figure 4, $L O D_{i+1}$ rendering number is less than $2 / 3 m^{2}$. When visible LOD number is $n$, total rendering grid numbers are: $S$ $=2+2 \mathrm{n} / 3 \mathrm{~m}^{2}$, when $n=5, S=$ $5.3 m^{2}$, if $m=64$, than $S=21,708$.

After using these strategies, all necessary rendering grid numbers have been cut down to feasible degree for animation. But for real-time animation this number

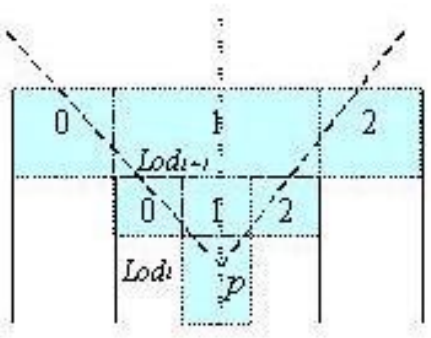

Fig. 4. LOD blocks cutting out by view field 
should not be bigger than 10,000 . Therefore, we need further processing which will be addressed in next section.

\subsection{Seam problem between two LODs}

During regular grid division process, different levels use different resample intervals. If we don't adapt further special processing, a seam would appear between two LODs. As seams appear on the sides of two adjacent LOD areas, and resample interval ratio of these two LOD areas is 2, we could change drawing one square to three triangles. Four sides need special

processing respectively. Figure 5 Fig. 5 . Filling gaps between two levels shows the processing.

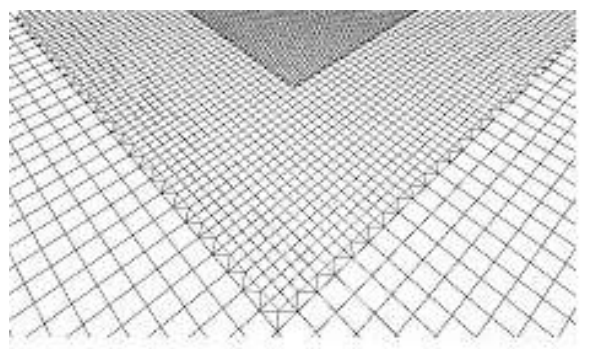

\section{Huge DEM data sets management and scheduling}

Before discussing visualization, we firstly need to manage the huge DEM and its corresponding image data sets. As many research works on huge raster data management have been done, we use following management method in our paper:

- Delaminate DEM and image data according its resolution and precision

- Each level has same resolution and precision

- Precision or resolution for different level increases principally in $2^{n}$;

In order to visualize DEM data in real-time, DEM and image data has to be divided into blocks. Blocks size should be $\left(2^{\mathrm{n}}, 2^{\mathrm{m}}\right)$. $(1024,1024)$ is the ideal size. DEM division and image division must be kept consistent.

Divided blocks are managed in DBMS using BLOB type according to their levels. One block has a record such as: serial number, row and column number, position in original raster data, corresponding spatial data and the block's raster data matrix;

An index file is established to save raster data according to their level structures. This index file is read into memory in real-time visualization process. The file manages reading from and releasing to each block data.

In real-time visualization process, it should permit observer to view terrain area both partially and fully. So the data scheduling need to switch DEM and image data between blocks and levels. The details are:

- Using view parameters calculate LOD areas and select visible DEM level. Then look up DEM and image data blocks in database and if blocks spatial area intersect with LOD areas, then read these blocks; 
- Judge each data block in memory whether it is still visible. If not, release memory occupied by this data block;

- As data blocks reading and releasing would affect DEM visualization continuity, it is needed to set a buffer with suitable size. In addition, visualization process and data access process should be processed in each program thread.

\section{Discussion}

Two key points need to be discussed.

\subsection{Problem relate to $L O D$ area size}

We have pointed out that the size of any $L O D_{n}$ could be expressed using $2^{n} m$. As resample interval of $L O D_{n}$ is $2^{n}$, rendering grid numbers of $L O D_{n}$ are decided by the value of $m$. At the same time, according to above scheduling, data blocks that need to be read into memory are determined by the value of $m$. For personal computer environment, the block numbers in memory need especially to be controlled carefully. From our experiment processes, we have examined $m$ value and found that 64 is an ideal value for $m$. When resample interval value is $2^{n} n=2,4,8, \ldots$, the sequence values of LOD area are 256, 512, 1024, 2048, ... When 5 LODs are used in visualization, three data levels are rightly need to be read at each time. If each level need eight blocks (DEM data blocks and its corresponding image data blocks), realtime rendering to three level data needs 24 data blocks to be read. Through cut off by view field (at least 50\%), real-time rendering grid number are $(64 \times 64+64 \times 32+$ $64 \times 32+64 \times 32+64 \times 32)=12288$.

\subsection{Problem of continuity of frame speed and visualization effect}

With the observer position changing, view field should also be changed; therefore, switches between LODs are needed. As different resolution levels in database are equal to dynamic delamination and dynamic delamination is calculated at real-time, LOD switch is the exchanges between two different resolution data levels. It is actually data reading and releasing process. One switch process might need reading eight data blocks including DEM and image data. This would greatly affect continuity of frame speed. In order to solve this problem, a buffer must be used to read these data blocks that would be rendered in recent frames. For high fly speed, this question is still there.

Figure 6 shows four visualization effects rendered using different resample interval at same place. It is found that visualization effects for resample interval value 8 and 1 are same for same image data. If we use 8 as the highest resample interval value, then value 12288 is reduced to 3072 . This value is really a widely acceptable value. Therefore, users can select between visual speed and effect from the system.

One obvious defect of our method is that many distortions would appear during animation process in sides of LOD areas. Such distortion is effected by terrain 
undulation. But distortion is not too much as it appears in far view areas, and it could be implemented by adding plants and fog effect.

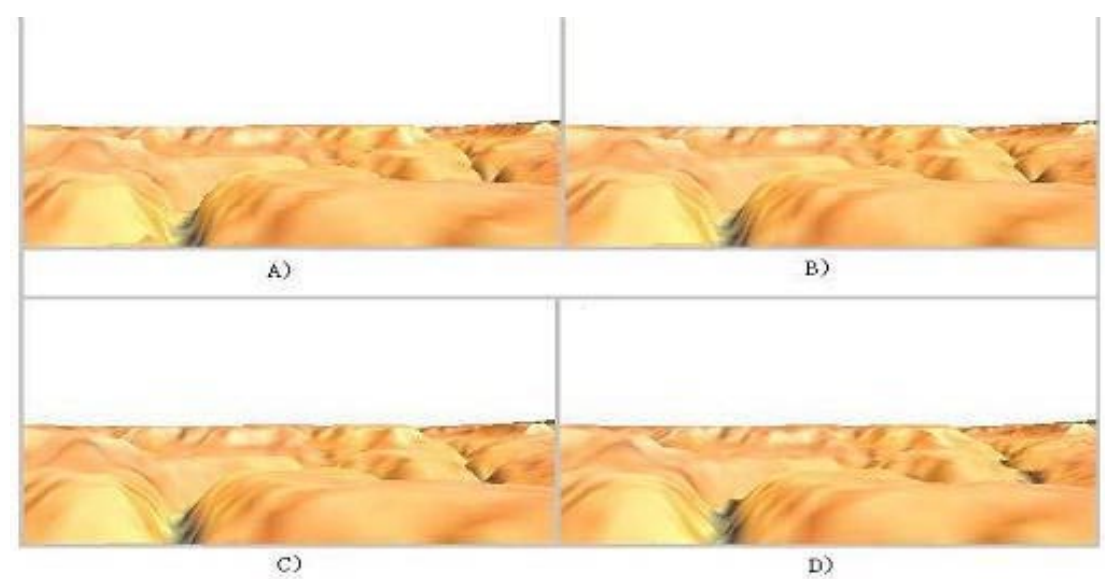

Fig. 6. Visualization effects using different sample intervals (Resample interval of A, B, C, D are $1,2,4,8$ respectively)

\section{Experiment}

As it is difficult to obtain a huge DEM data set we used a DEM data with block size $2048 \times 4096$ and its corresponding image block, which is downloaded from "ftp://ftp.research.microsoft.com/users/hhoppe/data/terrain/". We copied one DEM block $(8 \mathrm{Mb})$ and its corresponding image block $(24 \mathrm{Mb})$ into 64 copies. All DEM and image data blocks were divided into subblocks of size $1024 \times 1024$ (about $3 \mathrm{Mb}$ ), and stored into DBMS database. Its spatial position assigned according to blocks arrangement and blocks position in original data. We used resample interval sequence $2^{n}(\mathrm{n}=4,8,16 \ldots)$. The hardware configures of our PC are Window2000 professional, OpenGL 1.1, PIII CPU 667, RAM 128M, display card ELSA Erazor III LT, display memory $32 \mathrm{M}$. Real-time rendering speed is 20fps. Figure 7 shows five pictures rendering by the experiment system, which were copied from five different viewpoints.

\section{Conclusion}

In this paper, we developed a simple 3-dimensional visualization method. Comparing with existing methods, it has following merits:

- Much less calculation is used in real-time visualization process, so the visual process was not affected by the visualization calculation. 
- In addition to DEM and images data, few extra memories are used for visualization process. This is very useful for fly on terrain with a huge DEM data set;

- Regular grid expressed DEM model is easy to manage and visualize.

But for the different resample interval used to different LODs, terrain distortion would appear in sides of LOD areas. However, our method is much simple, and could be used to solve practice problem. It provides a feasible way for 3-dimensional realtime visualization of huge DEM data sets.

\section{Acknowledgments}

This publication is an output from the research project "Digital Earth" funded by Chinese Academy of Sciences and is partly supported by a grant from the Chinese National Sciences Foundations (No. 59904001). Also, Dr. Yong Xue should like to express his gratitude to Chinese Academy of Sciences for the financial support under the "CAS Hundred Talents Program" and "Initial Phase of the Knowledge Innovation Program".

\section{Reference}

1 Cignoni P., Puppo E., Scopigno R.: Representation and visualization of terrain surface at variable resolution, The Visual Computer, 13 (1997) 199-217

2 Fang T., Li Deren, Gong Jianya, Pi Minghong: Development and Implementation of Multiresolution and Seamless Image Database System GeoImageDB, Jorunal of WUHAN Technical University of Surveying and Mapping, 24 (1999) p222

3 Hoppe H.: Smooth view-dependent level-of-detail control and its application to terrain rendering, IEEE Visualization (1998), 35-42.

4 Kofler M.: R-trees for Visualizing and Organizing Huge 3D GIS Databases, [Ph.D. Dissertation], Graz Technischen Universität Graz, (1998)

5 Lindstrom P., Koller D., Ribarsky W., Hodges L.F., Faust N.: Real-Time, Continuous Level of Detail Rendering of Height Fields, [In] Proceedings of ACM SIGGRAPH 96, (http://www.cc.gatech.edw lindstro), (1996), 109-118.

6 Nebiker, S.: Spatial Raster Data Management for Geo-Information Systems-A Database Perspective, PhD Dissertation, Swiss Federal Institute of Technology Zurich (1997).

7 Wang H.W., Dong S.H.: A view-Dependent Dynamic Multiresolution Terrain Model, Journal of Computer Aided Design and Computer Graphics, 12 (2000) 575-579 

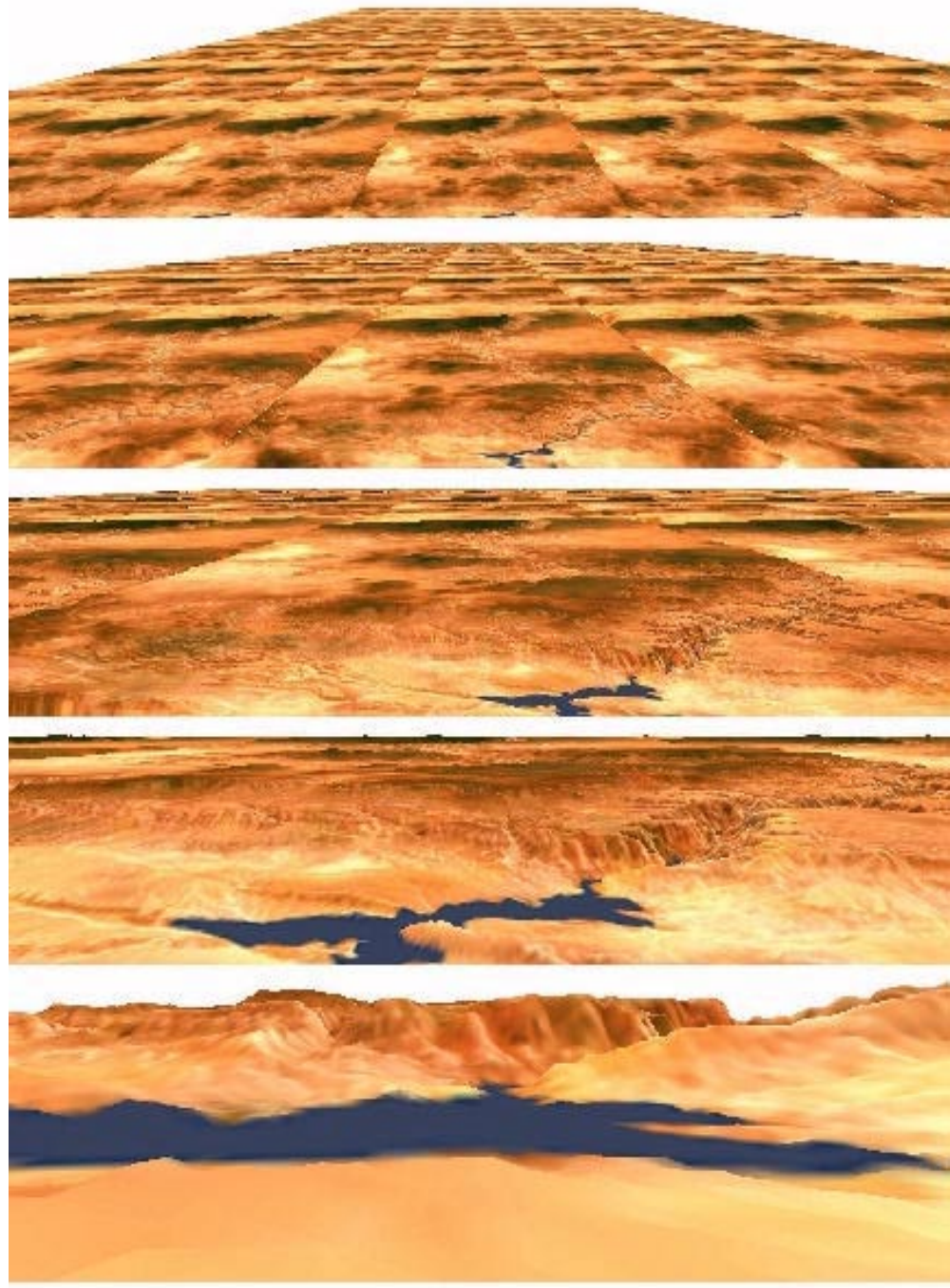

Fig. 7. 3D visualization of a terain landscape using the algonithm developed in the paper 